\title{
Oral Abstract Presentations at the 9th Canadian Conference on Dementia (CCD) Toronto, November 2017
}

http://dx.doi.org/10.5770/cgj.21.322

Capacity Planning for Community-Based Dementia Health Care Services in Ontario Using Administrative Health Care Databases and Agent-Based Simulation

D. Seitz ${ }^{1}$, T. Mahootchi², N. Warrick ${ }^{2}$, D. Shawcross ${ }^{2}$, A. Esensoy ${ }^{2}$. ${ }^{1}$ Queen's University; ${ }^{2}$ Cancer Care Ontario.

Background: Most individuals with dementia reside in community settings and this population requires a variety of supports and services to remain at home and maintain optimal quality of life. Capacity planning for dementia-related health services is important to understand the current needs of persons with dementia and plan for their future needs. Our project developed a Dementia Capacity Planning model for the province of Ontario in Canada to understand the current and future numbers of individuals with dementia in Ontario and anticipate their health service utilization.

Methods: A validated diagnostic algorithm was used with provincial administrative health care databases to identify the historical number of community-dwelling individuals with dementia in Ontario between 2010-2015. The historical patterns of health service utilization including home care services, and applications for long-term care (LTC) admissions were then determined. Agent-based simulation models were then used to simulate future numbers of community dwelling individuals with dementia in Ontario as well their future health service utilization based on demographic trends and current health system resources.

Results: The number of community-dwelling individuals with dementia increased from 81,637 individuals in 2010 to 106,427 individuals in $2015(+30 \%)$. Using conservative estimates, the simulation model projected that this number will increase to 143,557 by $2020(+35 \%$ over 2015$)$. The total number of individuals with dementia in the community who have requested admission to LTC and were awaiting placement increased from 12,866 in 2010 , to 22,063 in $2015(+71 \%)$ and this number is projected to increase to 39,789 individuals by $2020(+80 \%$ over 2015). The annual number of personal support worker hours provided by home care services is projected to be over 11 million hours by 2020, an increase of 50\% over 2015 utilization.
Conclusions: The growing population of older adults with dementia in Ontario will result in significant increased demands for home care and LTC services. Simulation models can be useful to understand these future demands and help in planning services accordingly. Significant changes to health services and supports for persons with dementia will likely be required to support this growing and vulnerable population in Ontario in the future.

Dementia, Including Alzheimer's Disease, in Canada: Prevalence, Incidence and All-Cause Mortality Trends from the Canadian Chronic Disease Surveillance System

C. Pelletier, S. Bartholomew, K. Sabou, L. McRae, J. Toews. Public Health Agency of Canada.

Background/Objectives: Dementia is a growing public health challenge in Canada, as acknowledged by the National Strategy for Alzheimer's Disease and Other Dementias Act. Monitoring the epidemiological burden and trends over time can generate key information to support public health actions. As of 2016, the Canadian Chronic Disease Surveillance System (CCDSS) supports the national surveillance of diagnosed dementia, including Alzheimer's disease. This presentation will summarize CCDSS data on dementia. Trends in the prevalence, incidence and all-cause mortality will also be presented.

Methods/Overview: The CCDSS is a pan-Canadian partnership, led by the Public Health Agency of Canada, that uses linked health administrative databases in all provinces and territories (except Saskatchewan for dementia) to collect data on more than 15 chronic conditions. A validated algorithm identified cases of diagnosed dementia among Canadian seniors (aged 65+) when they had:

- $1+$ hospitalization records or $3+$ physician claims within 2 years (minimum 30 days between each claim) with an ICD code for dementia; or

- $1+$ anti-dementia drug prescriptions. 
Prevalence, incidence and all-cause mortality data were disaggregated by sex, 5-year age group, and fiscal year. Annual average percent changes (AAPC) were estimated using log-linear regression models. The Wald chi-square statistic and associated $\mathrm{p}$-value were used for significance testing.

Results: Using the most recent data available, in 2013-2014, over 402,000 Canadian seniors (aged 65+) were living with diagnosed dementia (prevalence overall: 7.1\%; men: 5.6\%; women: 8.3\%), and 14.3 new cases were diagnosed per 1,000 senior population (incidence men: 12.4 per 1,000; women: 15.8 per 1,000 ). Prevalence and incidence estimates roughly doubled from one 5 -year age group to the next. Although estimates were similar between sexes in younger age groups, the differential tended to increase with age. The age-standardized all-cause mortality rate was approximately 4 times higher among seniors with versus without dementia. Between 2003-2004 and 2013-2014, there was a $21.2 \%$ relative increase in the age-standardized prevalence of diagnosed dementia (AAPC: $1.9 \%, p<.05$ ). The agestandardized incidence fluctuated during this 10 -year period. Data suggest a decline in incidence since 2009-2010, but the overall trend was not significant. During the same timeframe, the age-standardized all-cause mortality rate among Canadian seniors with dementia decreased (AAPC: $-2.0 \%, p<.05$ ), but not as much as the rate among Canadian seniors without dementia (AAPC: $-2.8 \%, p<.05$ ). While CCDSS data capture changes in population health status, they may also reflect changes in data collection methods, disease coding/classification systems, and clinical/billing practices over time. Additional work is needed to fully explore the contribution of potential data artefacts versus actual epidemiological variations.

Conclusions: CCDSS data will be used to monitor the epidemiological burden and trends of diagnosed dementia to help support the planning of health resources and the implementation of health policies, and evaluate future progress.

\section{Delivering Cognitive Stimulation with Technology}

A. Astell ${ }^{1}$, S. Smith ${ }^{2}$, S. Potter ${ }^{2} .{ }^{1}$ Ontario Shores Centre for Mental Health Sciences; ${ }^{2}$ University of Sheffield.

Background: Cognitive Stimulation Therapy (CST) (Spector et al., 2003) is a group-based activity for people with dementia using artefacts and music to prompt social interaction that has been shown to deliver significant improvement in cognitive function and quality of life. CIRCA (Computer Interactive Reminiscence and Conversation Aid) is an interactive, multimedia system to support conversation between people living with dementia and care partners (Astell et al., 2010). CIRCA is supplied with photos, music and videos, is easy to use and requires no preparation by staff. The potential of CIRCA to deliver cognitively stimulating group-based activities in day and long-term care settings has not previously been explored.

Method: One hundred and eighty people living with dementia are being recruited from 11 sites across the UK as part of a Horizon 2020 project. Half are using the existing standalone CIRCA device and half are using a new webbased version. Pre-, post- and three-month post-intervention primary outcome measure: Addenbrookes Cognitive Examination-III (ACE-III) (Hseih, 2013) (/100), with QOL-AD (Logsdon et al., 2003; scored between 13-52) and EQ-5D (/100) as secondary measures. All group sessions are being video-recorded to provide qualitative assessment of engagement, enjoyment and social interaction.

Results: The first 137 participants have been recruited; 84 $(12 \mathrm{M}, 72 \mathrm{~F})$ have completed the 8 -session programme and pre- and post-measures, of which 45 have also completed the three-month follow-up. These $45(7 \mathrm{M}, 38 \mathrm{~F})$ are aged between 69-97 years, 41 live in long-term care, with ACEIII scores at baseline between 6-84 (mean 48.3), QOL-AD scores between 21-41 (mean 31.24) and EQ-5D between 15-100 (mean 65.11). Preliminary analysis revealed a significant improvement in cognitive function over the three time points, $(p=.01)$, with significant increases from baseline to post-intervention $(p=.05)$ which was maintained at 3 -month follow-up $(\mathrm{p}=0.005)$. There was also a significant increase in quality of life scores over the three time points $(p=-.001)$.

Conclusion: Participation in the group sessions led to improved cognitive scores which were maintained at three months follow-up, along with improved quality of life similar to those reported for CST. The videos highlight the opportunities for enjoyment, engagement and social interaction. The data so far confirm the potential of CIRCA, an easy-to-use interactive multimedia system, as a cognitively-stimulating group-based activity for individuals in day and long-term care, including those with significant cognitive impairment (lower ACE-III scores).

\section{Responsive Behaviour: Meeting the Learning Needs of Inter-Professional Post-Secondary Health-care Students}

L. Schindel Martin ${ }^{1}$, P. Woo ${ }^{2,3}$, D. Cowan ${ }^{2,4}$, V. McLelland ${ }^{5}$, K. Newman ${ }^{1}$, D. Rose ${ }^{1}$, P. Miller $^{2}$, J.Ashbourne ${ }^{2}$, M. Ashley ${ }^{2}$, P. Julian ${ }^{1}$, M. McNee ${ }^{2}$, F. Zamora ${ }^{2} .{ }^{1}$ Ryerson University; ${ }^{2}$ McMaster University; ${ }^{3}$ Hamilton Health Sciences; ${ }^{4}$ St. Joseph's Healthcare, ${ }^{5}$ AGE Inc.

Background: Post-secondary healthcare students with limited knowledge to care for people experiencing the responsive, psychological and behavioural symptoms of dementia (BPSD) may avoid such individuals across healthcare sectors. The 
healthcare curriculum across disciplines is not standardized in terms of the principles for the recognition and management of BPSD. This insufficiency exists despite evidence that people cared for by professionals who recognize the underlying causes of BPSD and use communication strategies to validate their preferences and personhood, experience less behavior escalation. Students are requesting standardized dementia education programs to supplement their existing programs, but there are no such programs available in Ontario. Therefore, post-secondary institutions require accessible dementia education for their students.

Objectives: This study evaluated the implementation of the eLearning version of a dementia-specific educational intervention, Gentle Persuasive Approaches (GPA), with inter-professional health-care students at McMaster and Ryerson Universities. Participants completed an online GPA course and attended a Geriatrics Skills Day workshop both providing content about the application of responsive behaviour strategies.

Methods: This study used a mixed methods approach. Fiftytwo student volunteers completed standardized quantitative measures including: 1) self-efficacy, 2) competence and 3) caring scales, as well as 4) a multiple choice knowledge test, each administered pre- and post-intervention. Qualitative measures consisted of themes extracted from open-ended questions administered immediately pre- and post-intervention, as well as from a focus group conducted after the Skills Day workshop. The three main questions administered both online and within the focus groups were: Q1) What happens when you experience/witness episodes of BPSD in persons living with dementia? Q2) What would you like to learn more about with respect to expression/management of BPSD? Q3) What are the best practices that should be applied to persons with dementia experiencing BPSD?

Results: Participants demonstrated significant improvements in self-efficacy $(p<.001)$, competence $(p<.001)$, caring $(p<.001)$, and knowledge $(p<.001)$ in dementia care postintervention. Significant differences in self-efficacy across academic programs were observed prior to completion of GPA eLearning, but post-intervention self-efficacy scores were equivalent across programs. Qualitative findings revealed that, regardless of program, prior to GPA eLearning intervention students identified themselves to be unprepared to provide emotional support, interact using dementiaspecific communication strategies or effectively mitigate the risk associated with responsive behaviours. The emergent overarching theme reflecting pre-intervention responses was No Idea Where to Begin. Post-intervention, participants were able to describe, explain and demonstrate the application of dementia-specific communication, and interpersonal care and safety strategies. The emergent overarching theme reflecting post-intervention responses was Connecting with the Person.
Conclusions: Results provide evidence that the eLearning version of the GPA curriculum warrants broader dissemination through future studies within inter-professional postsecondary health-care student programs.

\section{Frailty Moderates the Relationship Between Alzheimer's Neuropathology and Cognition}

L. Wallace ${ }^{1}$, O. Theou ${ }^{1}$, J. Godin ${ }^{2}$, M. Andrew ${ }^{1,2}, \mathrm{~K}$. Rockwood ${ }^{1,2}$. ${ }^{1}$ Dalhousie University; ${ }^{2}$ Nova Scotia Health Authority.

Background: The neuropathological features of Alzheimer's disease (AD) are not always well correlated with the clinical presentation of dementia. However, dementia and frailty are closely linked: both are strongly related to advanced age and vulnerability to adverse health outcomes. It is possible that frailty interacts with neuropathological features of $\mathrm{AD}$ to increase vulnerability to cognitive impairment and dementia.

Objective: To examine if frailty moderates the relationship between $\mathrm{AD}$ neuropathology and cognition.

Methods: This was a cross-sectional analysis of data from the Rush Memory and Aging Project, a clinico-pathological study of older adults living in retirement communities in the USA. Participants were followed with annual clinical and neuropsychological evaluations and at time of death all had an autopsy. AD neuropathology was quantified using a measure of global pathology burden that was derived from counts of neuritic plaques, diffuse plaques, and neurofibrillary tangles as determined by microscopic examination of silver-stained slides from 5 regions: entorhinal cortex, hippocampus, midtemporal cortex, inferior parietal cortex, and midfrontal cortex. Frailty was operationalized using the deficit accumulation approach, with a frailty index constructed from 41 health variables including function, comorbidities, symptoms, and signs; the frailty index was binned in increments of 0.1 for regression analysis. Cognition was operationalized using the 30-item Mini-Mental State Examination (MMSE). Linear regression was used to test the relationship between frailty and neuropathology, and cognition after controlling for age, sex, education, APOE genotype. Process moderation model syntax was applied in SPSS to evaluate the moderation effect of frailty on the relationship between neuropathology and cognition.

Results: 635 adults $(83.3 \pm 6.0$ years, $67.2 \%$ female) were included in this analysis. At time of death, $52.8 \%$ suffered from AD-type dementia. Participants with dementia were significantly more frail $(\mathrm{F}=10.55, p<.001)$ and were more likely to have high neuropathological burden $(\mathrm{F}=33.36, p<.001)$ than 
those without dementia. Regression analyses demonstrated that both frailty and global pathology were independently associated with cognition (Odds Ratio [OR] $=-1.87, p<.001$; $\mathrm{OR}=-5.11, p<.001$, respectively), and that their interaction was also significant $(\mathrm{OR}=-0.79, p=.003)$. When probed, the interaction demonstrated a moderating effect of frailty on the relationship between neuropathology and cognition such that as frailty increased, the relationship between neuropathology and cognition strengthened (mild frailty $\mathrm{OR}=-3.78$, $p<.001$; moderate frailty $\mathrm{OR}=-5.09, p<.001$; severe frailty $\mathrm{OR}=-6.40, p<.001)$.

Conclusions: These findings suggest that in frailer people, the structural deficits (neuropathology) are more likely to impact function (cognition) than in less frail people. This is possibly due to a lack of systemic redundancy resilience, or increased vulnerability in the frail individual. Further research should assess longitudinal change to better elucidate this complex relationship. 